\title{
Eruptive nevi after burn injury
}

\author{
Ali Tareen ${ }^{1}$, Kristine Pallesen ${ }^{1}$, Tine Vestergaard ${ }^{1}$
}

1 Department of Dermatology and Allergy Centre, Odense University Hospital, Odense, Denmark

Key words: eruptive nevi, burn injury, traumatic nevi, EMN

Citation: Tareen A, Pallesen K, Vestergaard T. Eruptive nevi after burn injury. Dermatol Pract Concept. 2018;8(1):66-67. DOI: https://doi. org/10.5826/dpc.0801a16

Received: September 11, 2017; Accepted: October 24, 2017; Published: January 31, 2018

Copyright: @2018 Tareen et al. This is an open-access article distributed under the terms of the Creative Commons Attribution License, which permits unrestricted use, distribution, and reproduction in any medium, provided the original author and source are credited.

Funding: None.

Competing interests: The authors have no conflicts of interest to disclose.

All authors have contributed significantly to this publication.

Corresponding author: Ali Tareen, MD, Department of Dermatology, Odense University Hospital Kløvervænget 15, Indgang 142.5000 Odense C, Denmark. Tel. +45 51270721. Email: dr.alitareen@gmail.com

ABSTRACT Eruptive melanocytic nevi (EMN) is an unusual phenomenon characterized by the abrupt development of multiple melanocytic nevi over weeks to months in association with an underlying trigger. The underlying mechanisms are not fully understood, however, they have been associated with a variety of conditions. EMN is relatively uncommon and might be underreported due to the absence of close monitoring, insufficient recognition, and the presumed benign course of the condition. We describe the first case report of acral EMN associated with a burn wound on a 2-year-old child. Familiarity is important to differentiate EMN from neoplasms.

\section{Introduction}

An "outbreak of pigmented moles" was first described by Hutchinson in 1868 , in a 22 -year-old healthy woman. Although there is no current clear definition of eruptive melanocytic nevi (EMN), it generally describes the abrupt development of multiple melanocytic nevi over weeks to month in association with an underlying trigger [1].

\section{Case Report}

A 2-year-old child with Fitzpatrick skin type II was referred by her general practitioner because of abrupt development of pigmentation on her left palm. The child had accidentally burned her hand on a hotplate a half year prior to the consultation. The accident was presumably a mixture of first- and superficial second-degree burns.

Initially the patient developed small reddish macular papules on the left palm, which over the next couple of months became pigmented (Figure 1). Prior to the accident, a congenital nevus on the palm near the proximal interphalangeal third and fourth digits had been observed. The patient had otherwise only a few nevi on the body.

Dermatoscopy revealed an abundant number of $1-4 \mathrm{~mm}$ melanocytic nevi with light and dark brown colors, symmetrically arranged globules and dots at the periphery, and with a parallel furrow pattern (Figure 2).

The patient's nevi are being monitored for further development.

\section{Discussion}

There are no clear predisposing factors for the development of EMN, though a variety of associations have been proposed. There has been an association in cases of Stevens-Johnson syndrome/toxic epidermal necrolysis, erythema multiforme, bullous pemphigoid, eczema herpeticum, and exposure to sulfur mustard gas. There has also been an association with 


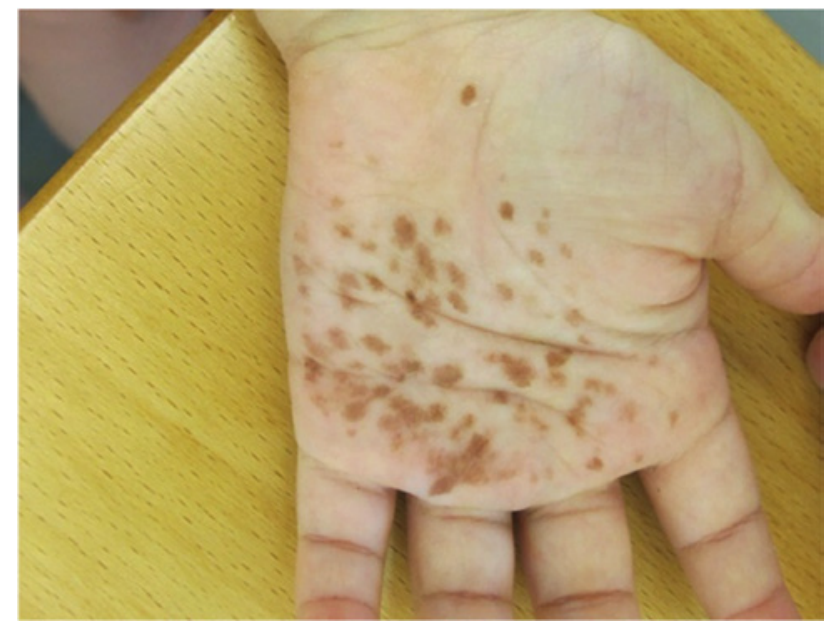

Figure 1. Eruptive nevi on the palm 6 months after burn injury. [Copyright: @2018 Tareen et al.]

conditions leading to compromised immunity, such as biologic and non-biologic immunosuppressants, biologic and non-biologic chemotherapeutics, malignancy, and AIDS. EMN has also been linked to Addison's disease, cutaneous mastocytosis, melanocyte stimulators, insulin, PUVA, and Koebner phenomenon [1-8]. The relevant differential diagnoses include postinflammatory melanosis and idiopathic eruptive macular pigmentation, which typically presents as non-confluent macules on the face, neck, proximal extremities, and trunk without preceding inflammatory lesions or drug exposure [9]. The dermoscopic presence of globules and dots make these diagnoses less likely.

Nevus spilus typically has a larger background patch with more pigmented macules within the borders and a stable appearance. There was no pigmented background patch in our case, and the pigmented pattern had an eruptive appearance. It is not known whether the close proximity to a congenital nevus, as in this case, plays a role in the development of EMN. This has not been described in the literature, to the best of our knowledge, and we do not believe that there is a causative connection. Congenital nevi have a steady growth during early childhood [10]. The abrupt presence of melanocytic nevi with an underlying trigger makes the diagnosis of EMN likely.

Some authors believe that the common denominator is precipitated in genetically predisposed individuals. EMN has been linked to mutation in the BRAF V600E gene, which contributes to the development of malignant melanoma [2,7]. Association between EMN and malignant melanoma has not been described. Familiarity is important to differentiate EMN from neoplasms.

EMN has a strong predilection for the palmoplantar skin, although it has been described in other distributions [2]. This is different from sporadic melanocytic nevi that appear less frequently at acral sites. A hypothesis to support this could be that eccrine glands have a higher amount of melanocortin5 -receptors that bind melanocyte-stimulating hormone,

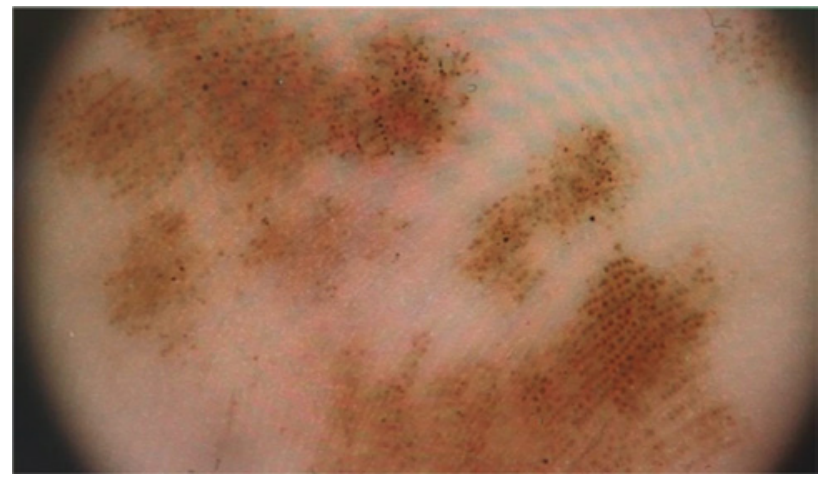

Figure 2. Dermoscopic view of eruptive nevi on the palm after burn injury. [Copyright: @2018 Tareen et al.]

which could contribute to the proliferation of melanocytic cells on the palmoplantar skin [11].

The small number of cases suggests that it is a relatively uncommon phenomenon, however, EMN might be underreported due to the absence of close dermatologic monitoring, insufficient recognition by practitioners, and the presumed benign course of the condition. This case is unique because this is the first described case of acral eruptive nevi associated with a burn wound.

\section{References}

1. Bovenschen $\mathrm{HJ}$, Tjioe $\mathrm{M}$, Vermaat $\mathrm{H}$, et al. Induction of eruptive benign melanocytic naevi by immune suppressive agents, including biological. Br J Dermatol. 2006 May;154(5):880-884.

2. Perry BM, Nguyen A, Desmond BL, Blattner CM, Thomas RS, Young RJ. Eruptive nevi associated with medications (ENAMs). J Am Acad Dermatol. 2016;75:1045-1052.

3. Navarini AA, Kolm I, Calvo X, et al. Trauma as triggering factor for development of melanocytic nevi. Dermatology. 2010;220:291-296.

4. Yoshida Y, Yamada N, Adachi K, Tanaka M, Yamamoto O. Traumatized recurrent melanocytic naevus with typical starburst pattern on dermoscopy. Acta Derm Venereol. 2008;88(4):408-409.

5. Schulze F, Erdmann H, Hardkop LH, et al. Eruptive naevi and darkening of pre-existing naevi $24 \mathrm{~h}$ after a single mono-dose injection of melanotan II. Eur J Dermatol. 2014;24(1):107-109.

6. Colson F, Arrese JE, Nikkels AF. Localized eruptive blue nevi after herpes zoster. Case Rep Dermatol. 2016;8(2):118-123.

7. John JK, Smalley KS. Identification of BRAF mutations in eruptive melanocytic nevi: new insights into melanomagenesis? Expert Rev Anticancer Ther. 2011;11(5):711-714.

8. Levy RM, Ming ME, Shapiro M, et al. Eruptive disseminated Spitz nevi. J Am Acad Dermatol. 2007;57:519-523.

9. Dedhia A, Someshwar S, Jerajani HR. Idiopathic eruptive macular pigmentation: What is it actually? Int J Dermatol. 2015;54(12):1462-1465.

10. Rhodes AR, Albert LS, Weinstock MA. Congenital nevomelanocytic nevi: proportionate area expansion during infancy and early childhood. J Am Acad Dermatol. 1996;34(1):51-62.

11. Woodhouse J, Maytin EV. Eruptive nevi of the palms and soles. $J$ Am Acad Dermatol. 2005;52(5 Suppl 1):S96-S100. 